\title{
Diabetes mellitus in a patient with Leri-Weill dyschondrosteosis
}

\section{Didem Ozdemir ${ }^{1}$, Șefika Burçak Polat ${ }^{1}$, Nagihan Beştepe ${ }^{1}$, Saliha Ozbek²${ }^{2}$, Reyhan Ersoy ${ }^{1}$, Bekir Cakir ${ }^{1}$}

${ }^{1}$ Yildirim Beyazit University, School of Medicine, Ataturk Education and Research Hospital, Department of Endocrinology and Metabolism, Ankara, Turkiye

${ }^{2}$ Yildirim Beyazit University, School of Medicine, Ataturk Education and Research Hospital, Department of Internal Medicine, Ankara, Turkiye

\section{Introduction:}

Leri-Weill dyschondrosteosis (LWD) is a rare autosomal dominant dyschondrosteosis characterized by Madelung deformity and mesomelic dwarfism. Majority of cases are associated with haploinsufficiency of the short-stature homeoboxcontaining (SHOX) gene. Here we report a patient with LWD and diabetes mellitus (DM).

Case Report:

A 31 years old man applied with polyuria and polydipsia. He did not have any chronic disease. In family history, father had type 1 and mother had type 2 DM. His height was $146 \mathrm{~cm}$ and weight was $62 \mathrm{~kg}$, with a body mass index of $29.1 \mathrm{~kg} / \mathrm{m}^{2}$. Laboratory investigation revealed a fasting blood glucose of 298 $\mathrm{mg} / \mathrm{dl}, \mathrm{HbAlc} 13.4 \%$, insülin $8 \mu \mathrm{U} / \mathrm{ml}$ (2.6-24.9), cpeptide $1.65 \mathrm{ng} / \mathrm{ml}$ (0.1-3.6). AntiGAD, anti-insulin and islet-cell antibodies were negative. Intensive insulin treatment was started. The result of genetic analysis made to determine cause of short stature about 1,5 years ago was 45,X,-Y[5]/46,X,der(Y)t(Y;Y)(p11.2;q11.21).

There was deletion in the SHOX gene of Y chromosome. He had bilateral Madelung deformity which is an epiphyseal growth plate disturbance characterized by shortened and bowed radii and ulnae leading to dorsal dislocation of the distal ulna (Figure 1).Thus, he was diagnosed to have LWD.
Figure 1: Bilateral Madelung deformity of wrists

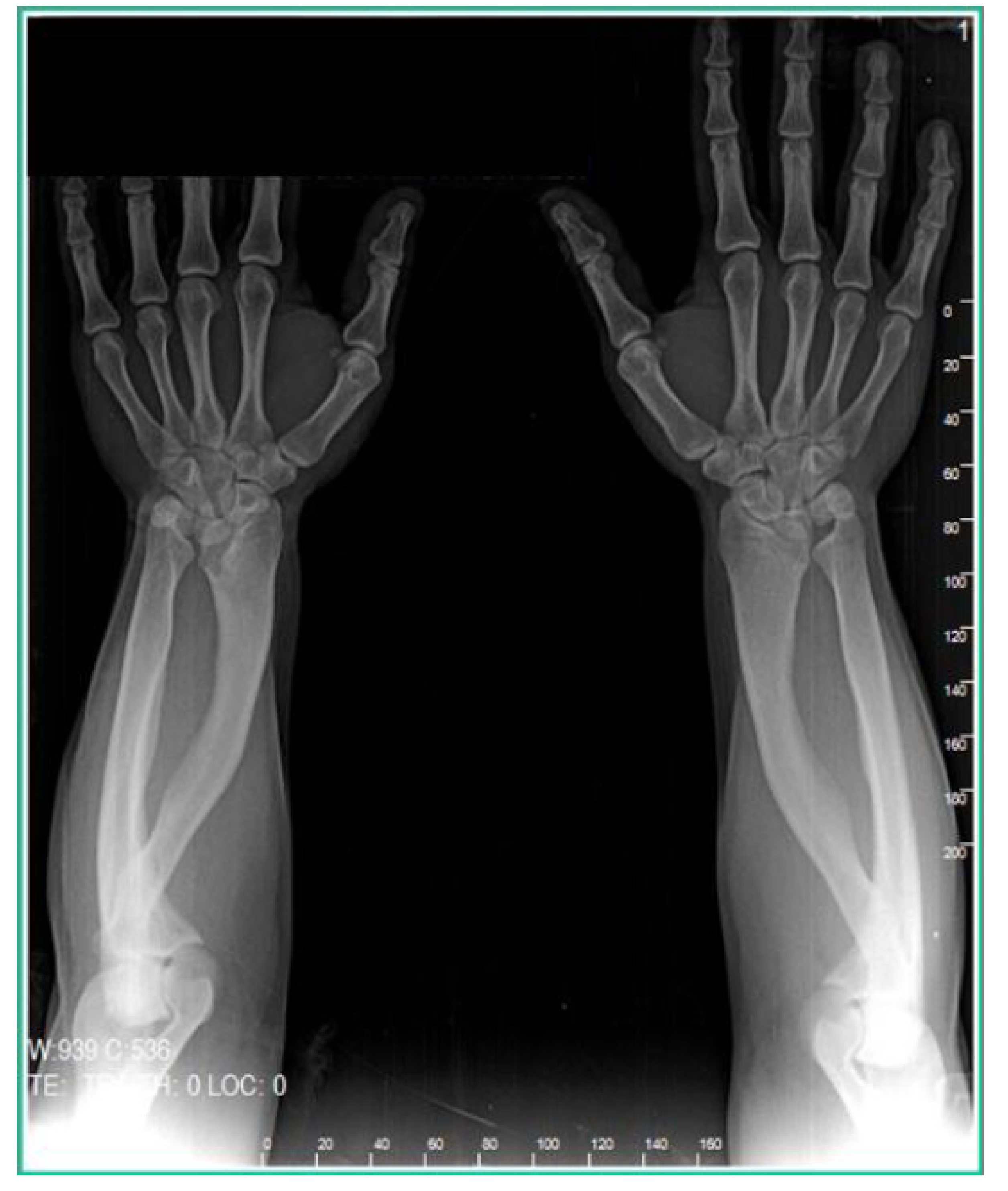

\section{Conclusion:}

Haploinsufficiency of the SHOX gene which is located on the pseudoautosomal region of both the $\mathrm{X}$ - and $\mathrm{Y}$ chromosomes is a cause of short stature in various clinical conditions including LWD. There are previous reports indicating inverse association between height and diabetes, and showing increased prevalance of gestational $\mathrm{DM}$ in patients with short stature. In the literature, clinical features of LWD is limited to skeletal anomalies and short stature. This is the first report of DM in a patient with this syndrome. Although, it is probable that this might be just a coincidence, we think possible association between impiared glucose metabolism and syndromes related with short stature needs further investigation. 